\title{
Eating with a Conscience: Toward a Visual and Contextual Nutrition Facts Label
}

\author{
Darius Coelho * \\ Stony Brook University
}

\author{
Helen $\mathrm{He}^{\dagger}$ \\ Stanford University.
}

\author{
Maxim Baduk \\ Cornell University.
}

\author{
Klaus Mueller ${ }^{\S}$ \\ Stony Brook University
}

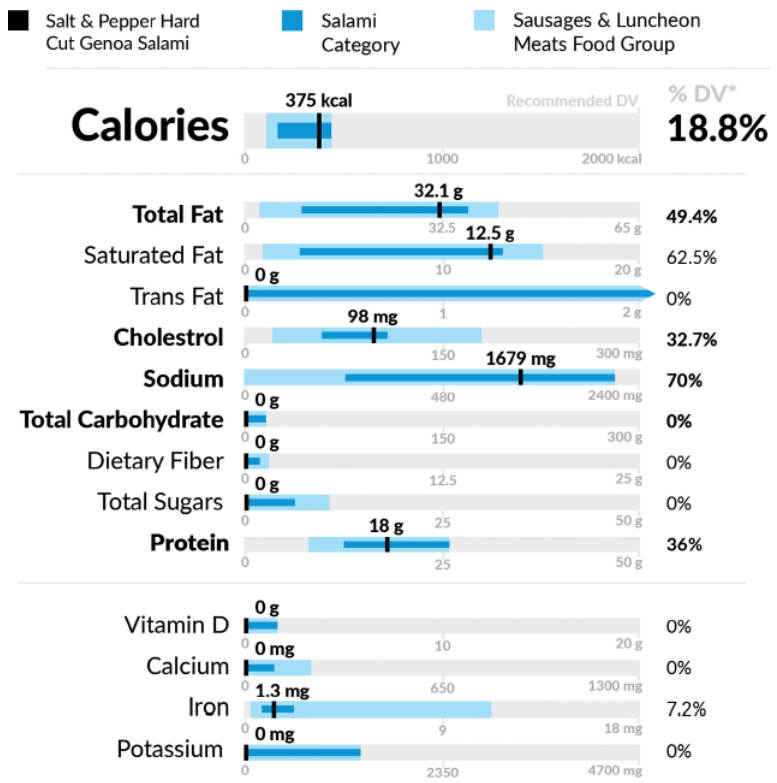

(a)

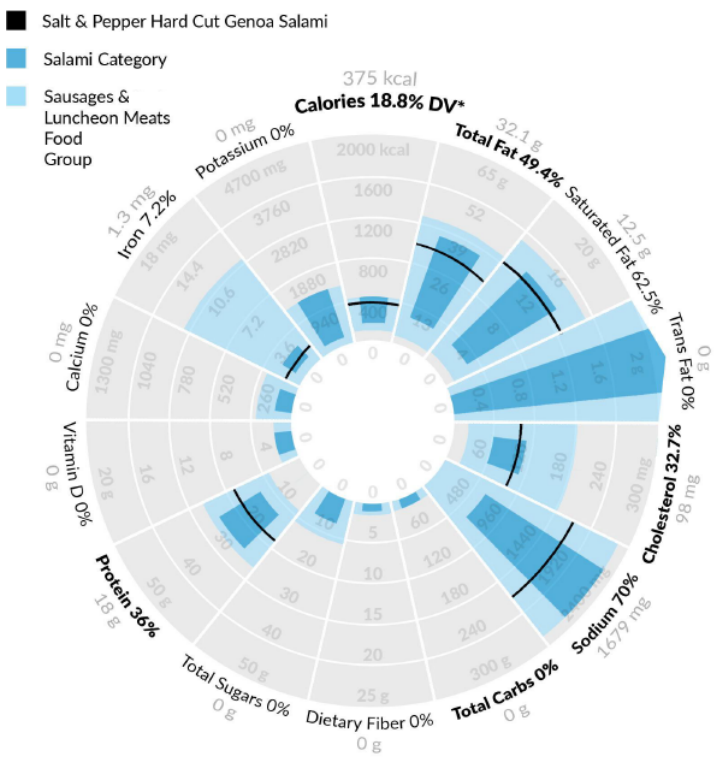

(b)

Figure 1: Examples of our linear (a) and radial (b) visual nutrition facts label designs based on the bullet graph. Both labels show the nutritional content of the food 'Salt \& Pepper Hard Cut Genoa Salami' as well as the range of nutrient levels for all foods in the 'Salami' category (dark blue) and the 'Sausages \& Luncheon Meat' food group (light blue).

\section{Abstract}

The large variety of food products available in today's market is making it increasingly difficult for the diet-conscious consumer to select the appropriate foods to maintain a balanced diet. To assist consumers, we design a visual nutrition facts label that provides the viewer with a quick overview of a food's nutritional content while presenting them with contextual information that compares it with similar foods. Our core design is based on the bullet graph as it is capable of displaying the level of a nutrient in a food product along with the range of nutrient levels in other similar foods thus providing a context. In our work, we create multiple design variations of our visual nutrition labels based on the bullet graph and test which design is the most preferable and effective.

Index Terms: Human-centered computing_VisualizationVisualization application domains-Information visualization Human-centered computing-Visualization-Visualization design and evaluation methods

\footnotetext{
*e-mail: dcoelho@cs.stonybrook.edu

†e-mail: helenahe@stanford.edu

†e-mail: mb2474@cornell.edu

§e-mail: mueller@cs.stonybrook.edu
}

\section{INTRODUCTION}

Today, people are increasingly conscientious about maintaining a balanced diet and staying healthy; thus they are very careful in selecting food products. However, the current market offers consumers a large number of food products with varying levels of nutrients that make it difficult for people to make the most suitable choices. Some of these products are very similar in terms of appearance and taste but vary greatly in terms of their nutrients, and this can only be learned by reading the nutrition facts labels, or nutrition label for short, on the product. In the United States, the Food and Drug Administration (FDA) regulates the design of the nutrition label and constantly works on improving its design to improve comprehension. However, the current design is still difficult for a large number of consumers to understand and make comparisons between food products [12]. Particularly, people tend to make mistakes when performing computations and estimating the contribution of a food product to their daily nutrient requirement. Additionally, people tend to confuse numbers displayed on labels, for example they inappropriately read the percent daily value information instead of the actual amount of a nutrient. Our work aims to improve the current label design with visualizations.

In this work, we design a visual nutrition label to provide the viewer with an intuitive representation of the nutritional content of a food product as well as contextual information about how the product compares with similar food products. Specifically, our label is designed to indicate the quantity or level of individual nutrients in a food product while indicating where this quantity is placed within the 
range of quantities of this nutrient in similar products. For example, we report the quantity of protein in a chicken breast while also showing the range of the protein content across all poultry products as well as meat products. An example is shown in figure 1. This representation allows a viewer to quickly read the nutrient quantities as well as see if there exist alternatives with a higher or lower nutrient content. Additionally, we hypothesize that viewers would be more accurate at computations using the visual representations. In the remainder of this paper, we describe our design choices as well as design variations. We conduct a preliminary study to test the user preference and performance of our design variations and compare them to the standard nutrition label.

\section{Related Work}

Organizations such as the FDA regulate the design of the nutrition label so as to ensure general comprehension. However, studies show that even in its current form, the nutrition label is still confusing to a large part of the population primarily due to people misapplying the serving size, confusion due to extraneous material on the food label, and incorrect calculations [12]. Nutrition researchers who have studied how people read the current label [7] suggest that the current design be improved to engage the users while conveying numeric information in a simpler manner [11].

Nutrition tools such as MyFitnessPal [2], Fooducate [1], and MyPlate Calorie Counter [3] attempt to solve this issue with mobile apps that compute the nutrient contents of foods the user inputs. However, the study and use of visualization to communicate information displayed on the nutrition label is limited. Bayu et al studied the use of gauges in an augmented reality setting to visualize the nutritional content of food [4]. Riehmann et al designed and studied glyphbased characters to communicate the nutritional effects of foods to children [10]. Pratt [9] and Mah et al [8] go further by allowing users to compare food items. Pratt makes use of scatterplots with a color-coded background to compare the levels of two nutrients in multiple foods [9]. Mah et al develop an entirely new visual representation they call a Fingerprint that allows users to compare the nutritional content of any two foods [8]. While these designs have shown to be useful, they are unsuitable candidates as a replacement for the current nutrition label as they deviate substantially from the current design. In our work, we attempt to maintain the core design guidelines provided by the FDA while adding a visual element to the nutrition label.

\section{LABEL DESIGN}

Our goal is to create a visual nutrition label that allows general consumers to easily read a food's nutrient levels, see where this food stands within the range of similar foods, and compare it to other similar foods. For example, if a person is looking at a package of cheddar cheese, he or she should be able to quickly retrieve the nutritional contents of the cheese, see where these nutritional levels stand within the range of other cheeses as well as compare it to an alternative, say provolone. Thus, our visual label must provide the user with the ability to quickly and easily read both exact values as well as ranges while minimizing the space it occupies. We describe elements of our label design in the following subsections.

\subsection{The Data}

In order to provide a context or range of nutrient levels for similar foods, we make use of the U.S. FDA database of food. This database contains a list of most food products sold in the United States along with the nutrient levels present in each food. The FDA also categorizes the foods into broad categories and sub-categories. For each category and subcategory, we pre-compute the upper bound and lower bound of all the nutrient levels in a single serving of food within the category. This creates a range for each nutrient within different food categories and sub-categories. We then use these ranges while creating the visual label for a single food item by displaying the range of the nutrient for that food category and subcategory.

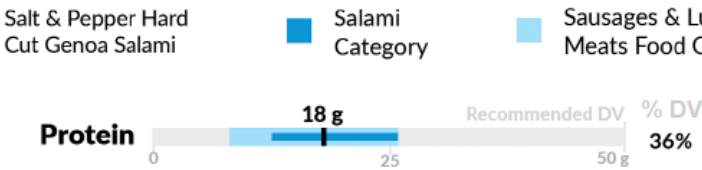

Figure 2: The level of a single nutrient, in this case protein of the food 'Salt \& Pepper Hard Cut Genoa Salami' shown with our bullet graph design. The representation also shows the range of protein content in the 'Salami Category' (dark blue) and in the parent food group 'Sausages \& Luncheon Meats' (light blue)

\subsection{Core Design}

We chose to model our label after the bullet graph developed by Stephen Few [6]. The bullet graph features a single quantitative measure along with complementary measures to which the featured measure can be related or compared. In addition, the graph reduces clutter with its small footprint and also reduces the cognitive load on the viewer [14]. This is ideal for our application as we want to enable the consumer to easily determine where a product's nutrition levels are situated within the range of nutrition levels of similar products. Additionally, the bullet graph design is based on bar charts and has some resemblance to progress bars which we believe would make it easily understandable to the general public.

For our application to food labels, we make minor modifications to the original bullet chart. An example applied to a single nutrient (protein) in a food product is shown in figure 2. Here we show the level of protein (featured value) for the product 'Salt \& Pepper Hard Cut Genoa Salami' that is listed under the 'Salami' category which in turn is under the 'Sausages and Luncheon Meat' food group.

The first modification to Few's original graph is that instead of using a bar to represent the featured value, we use a black vertical line marker. We chose this design as viewers may confuse a bar to be representative of a range instead of a single value. Additionally, in order to fit multiple bullet graphs into a food label, we need to use narrow bullet graphs thereby making it difficult to fit three bars (one for the featured value and two for the ranges) into the graphs. Second, we limit the extent of the range of values shown by a single bullet graph to the recommended daily value of the nutrient required by an average human. Nutrient levels or ranges that exceed the daily recommended value are indicated with an arrow toward the end of the graph as shown in the Trans Fat nutrient in figure 1a. This choice was based on the fact that studies [13] show that it is easier for users to compare the required daily quantity of a nutrient in terms of a percentage of the required daily amount rather than the actual quantity. Our design communicates both, the actual quantity through the line marker label (here, $18 \mathrm{~g}$ ) and the percentage based on the position of the marker within the bar as well as a label at the right end of the bar ( $18 \mathrm{~g}$ is $36 \%$ of the recommended daily protein diet of $50 \mathrm{~g}$ ).

We also show two complementary ranges using the light and dark blue bars, also shown in figure 2 . The light blue bar corresponds to the range of the nutrient values for all products in the same category of the featured product and the dark blue bar corresponds to a subcategory. By displaying both ranges, the user is more informed if she decides to look for an alternative. The ranges would inform her if she needs to look for an alternative in the same subcategory, category, or look outside of the category based on her dietary needs.

\subsection{Layout}

Foods contain multiple nutrients each of which we represent with a bullet graph. However, there is a limited amount of real estate 


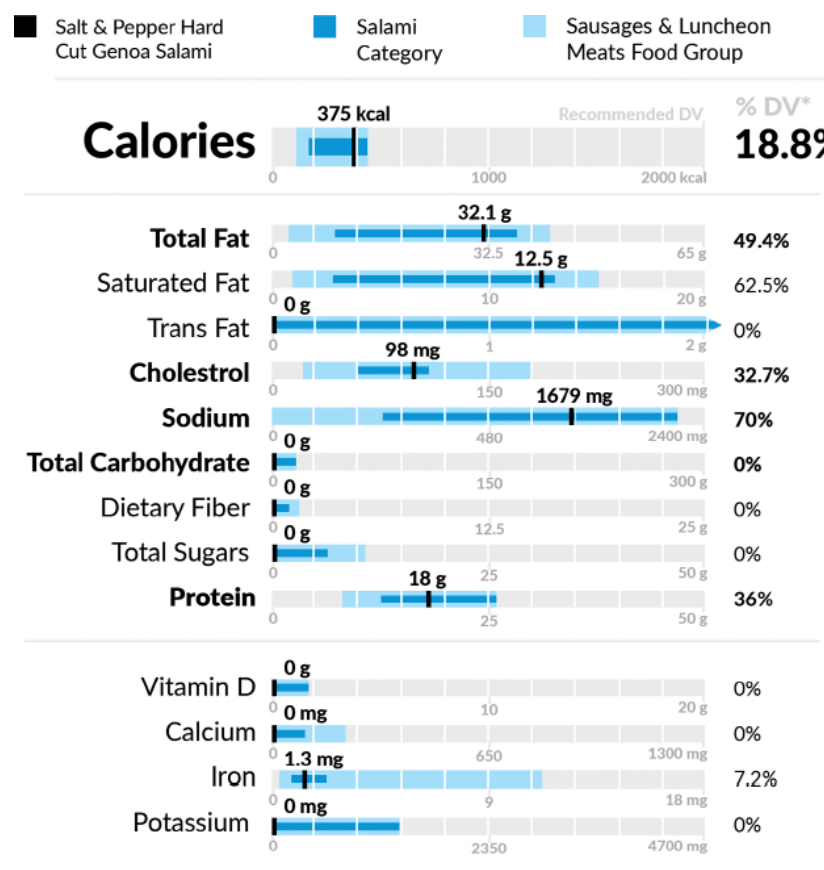

Figure 3: The linear nutrition label design with the discrete unit encoding. Here each bullet graph has been divided into 10 discrete units where each unit represents $10 \%$ of the required daily value of a nutrient.

available for food labels on food packages. Thus, in order to optimize the space occupied by the visual food label, we consider two layouts - linear and radial. Examples of the layouts are shown in figure 1.

In the linear layout, the bullet graphs are arranged vertically from top to bottom as shown in figure $4 \mathrm{a}$ and $4 \mathrm{~b}$. The nutrients are arranged from top to bottom in the order specified by the U.S FDA. We also follow the font-size specifications provided by the U.S. FDA. As the FDA specifies that the calorie font-size should be larger than the other nutrients, we increase the thickness of the bullet graph for calories proportionally.

In the Radial layout, the bullet graphs are arranged in a radial fashion as shown in figure $4 \mathrm{c}$ and $4 \mathrm{~d}$. Again the nutrients are arranged in the order specified by the U.S. FDA. But in this case, the first item, calorie count, starts at the $90^{\circ}$ or 12 o'clock position. In this arrangement, we choose to offset the start of each bullet graph from the center of the circle so as to avoid confusion for very low levels of nutrients. Here we follow most of the FDA's font specifications but only relax it marginally when it comes to calories. While this arrangement can save space as the number of nutrients increase, we believe it will be harder to read.

\subsection{Unit Encoding}

In addition to laying out the bullet graphs in different ways, we also implement different unit encodings. We use the traditional continuous encoding as shown in figure 1 and we used a discretized encoding shown in figure 3 . The continuous encoding represents the range of the nutrient values as a continuous bar while the discretized encoding divides the bar into ten individual segments each representing $10 \%$ of the range. We expect that the discrete encoding will help users to easily approximate the quantity of a nutrient in terms of the percentage of the daily recommend value as well as help users easily compare products. For example, a viewer reading the protein content of the salami in figure 3 can quickly see that the level is marked somewhere near the center of the fourth segment indicating that this product fulfills $35 \%$ of her daily protein requirement. Now she would have to look for other foods with a protein content of six and a half segments to complete her daily protein requirement.

\section{Pilot Studies}

We conduct a pilot evaluation of our visual nutrition labels with two studies. First, we test which layout is more preferable. We then test which unit encoding of the bullet graphs was beneficial. We also compare the standard text-based label to our design in both studies.

\subsection{Linear vs Radial}

The performance of reading values and ranges on linear and radial designs has been well studied by Brehmer et al [5]. Although the radial designs have shown to have limitations in terms of performance, it is important for us to learn which layout is preferred by general users as we expect a more preferable layout would be more engaging. In this study, we compared our linear label design, radial label design as well as the standard nutrition label. We created two additional labels which were variations of our visual labels; the variation only changed the location of the labels that indicate the percent daily required value for the nutrients. We also chose to remove the color as we did not want users to be engaged by color when comparing the visual labels to the standard black and white color. These are shown in figure 4 . We recruited 20 participants, 10 males and 10 females, for this study. All participants were students and were recruited at our university campus. The participants first received a brief introduction explaining each label design. Next, they were presented with a random ordering of all five designs and asked them to rearrange them based on their preference. Participants

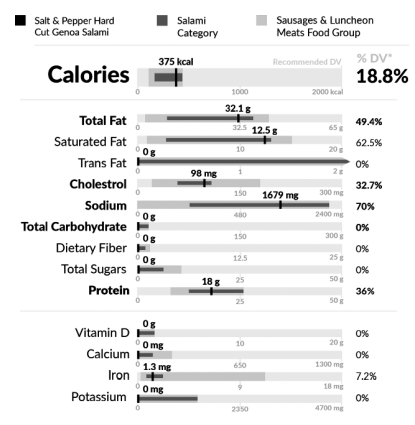

(a)

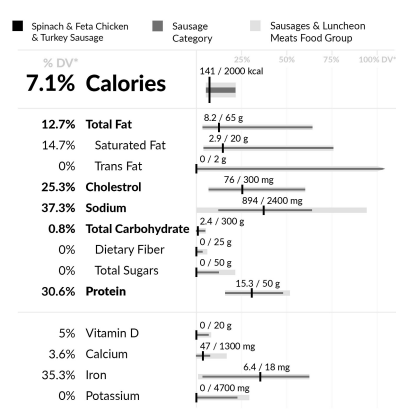

(b)

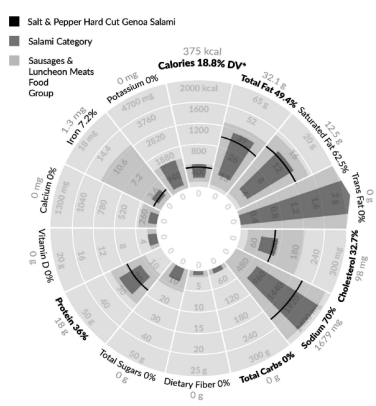

(c)



(d)

Figure 4: The label designs used in our preference study. Figures (b) and (d) differ from (a) and (c) respectively in terms of label placement of the percentage of daily value and the background of the bullet graphs. 


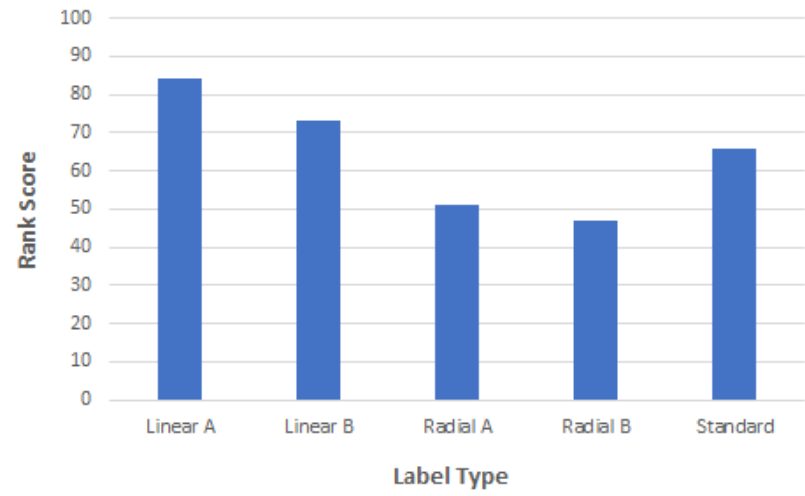

Figure 4: This graph shows the results of our preference test. The result show that the linear design Linear $A$ and Linear $B$ shown in figure $4 \mathrm{a}$ and $4 \mathrm{~b}$ respectively are more preferred than the radial designs Radial $A$ and Radial $B$ shown in figure $4 \mathrm{c}$ and $4 \mathrm{~d}$ as well as the standard nutrition label.

eventually submitted a list of labels ranked from most preferred to the least preferred. Each participant performed one ranking and the food represented by the label was selected randomly from three candidates.

The results are shown in figure 4 . The chart shows a rank score for each label type. The score was calculated by assigning a value of 5 if a participant gave a label the highest possible rank and a value of 1 for the lowest possible rank with intermediate ranks receiving values decreasing from 5 to 1 . Our results show that both the linear designs received higher rank scores than the standard label with the label shown in figure $4 \mathrm{a}$ receiving the highest score. On the other hand, both radial designs received lower rank scores than the standard label. Thus we conclude that the linear design clearly wins over the standard label in terms of user preference.

\subsection{Continuous vs Discrete}

Having learned that users prefer the linear design over the radial design, we decided to test the unit encoding performance only on the linear design. In this study, we compared the standard food label to the continuous linear design and the discretized linear design. For this study, we recruited 15 participants, 9 males and 6 females, ages 17 to 50. Participants were recruited via social media and had education levels varying from High School to Ph.D. Each participant answered questions using one of the three label designs applied to two randomly selected food products. Participants were asked a total of 13 questions per food product, divided into the categories: reading nutritional values (4 questions), reading nutritional ranges (5 questions), comparing values between two products ( 2 questions), and comparing ranges between two products ( 2 questions). These questions are replicated from those used in the study designed by Rothman et al [12] and shown in the supplementary material. Additionally, we asked participants who received the visual labels if they preferred it over the standard food label. Prior to answering these questions, participants received a brief introduction explaining each section of the label and how to read them. Then they were given 6 questions to which the answers were shown to familiarize them with using the label.

The results are presented in table 1 . We tested for significant differences between the performance of users for each label type and question type with a two sample t-test. They show that when it comes to reading and comparing values of the levels of a single nutrient, participants perform significantly better with our visual labels with an accuracy increase of greater than $25 \%$ over the standard label. Furthermore, the effect of the discretized unit encoding was minimal with users performing marginally better $(t(8)=-0.58, \mathrm{p}>0.05)$ with the discretized unit encoding with an accuracy of $95 \%$ versus the 92.5\% accuracy with the continuous unit encoding. However, the participants seem to perform better at comparisons of values $(\mathrm{t}(8)=$ $2.45, \mathrm{p}<0.05)$ when using the continuous unit encoding versus the discrete encoding. When it comes to reading and comparing ranges, we only report results for the visual labels as the standard labels do not have the capability of reporting ranges. Again the performance difference between the participants using the continuous and the discretized unit encoding was marginal, with a difference of only $2 \%$ $(\mathrm{t}(8)=0.25, \mathrm{p}>0.05)$ when reading ranges and a difference of only $5 \%(\mathrm{t}(8)=-0.63, \mathrm{p}>0.05)$ when comparing ranges. This indicates that there is no significant difference in user performance when using either encoding. However, when we asked users if they preferred their respective visual designs over the standard label, more users said that they preferred the linear label with the discretized unit encoding over the standard label.

Table 1: This table shows results of the effect of unit encoding on the user performance when reading nutrient levels and ranges.

\begin{tabular}{lccc}
\hline Measure & Label Type & \\
& Standard & Continuous & Discrete \\
\hline Reading Values & $62.5 \%$ & $92.5 \%$ & $95.0 \%$ \\
Reading Ranges & - & $52.0 \%$ & $50.0 \%$ \\
Comparing Values & $65.0 \%$ & $100 \%$ & $85.0 \%$ \\
Comparing Ranges & - & $80.0 \%$ & $85.0 \%$ \\
Preference & - & $60.0 \%$ & $100 \%$ \\
\hline
\end{tabular}

\section{Discussion}

We designed a visual nutrition label based on the bullet graph and conducted pilot tests on two layouts and two unit encodings in these visual labels. Our pilot study indicates that people have a preference for the linear layout over the standard text-based label, while the radial layout was not preferred over the linear or standard nutrition label. On the other hand, when evaluating the unit encoding, we did not find any significant differences between the continuous or linear encodings. However, it should be noted that studies were preliminary and had a limited number of participants hence future evaluations may lead to new conclusions. We believe our results for the layout preference study will hold with an increase in the number of participants as the radial layout has been shown to be problematic in multiple studies. But in the case of the unit encoding, based on our observations of users performing the tasks in the study, we do expect to see some differences as the number of participants increase. We believe that participants may find it easier to read values with the discrete encoding as it breaks down the scale into 10 ten smaller units making it easier for the user to approximate the level of a nutrient. However, we believe that the discrete encoding will cause some problems when it comes to reading ranges. This is because the ranges typically start from a non-zero value and would start from somewhere within one of the 10 units. It is also likely that the end of the range ends somewhere within one of the 10 units. This would make it difficult for users to first compute the starting and ending points of the range and how many units it spans. Further comprehensive testing is required to confirm our hypotheses.

\section{CONCLUSION}

We introduce a new method for visualizing nutritional information based on the representation of data in a graphical format, with the goal of improving readability, ease of use, and understanding of consumers over the standard label. Our pilot studies show that our labels have the potential to improve users' understanding of a product's nutritional value relative to other products of its type, and 
in improving users' ability to read nutritional values and compare those values between products. The use of our design shows the potential for increasing consumer understanding of the foods they eat and thus may encourage improvement in their dietary choices. Further research is needed to assess the effects of implementation on dietary choices, as well as to expand the user study to include more participants from a greater range of backgrounds to test whether the improvement is applicable to a wider range of consumers. Our labels are also generalizable; our label design can be applied to other product types as well. For example, they can be used to display the specifications of cellphones and laptops while showing the range of specifications across alternate products. In the future, we plan to test our labels extensively on a wider audience as well as on a product type other than food.

\section{ACKNOWLEDGMENTS}

This work was supported in part by NSF grant IIS 1527200, NSF grant IIS 1941613 and MSIT Korea ICT Consilience Creative program (IITP-2020-2011-1-00783).

\section{REFERENCES}

[1] Fooducate. https://www . fooducate. com/. Accessed: 2020-07-10.

[2] Myfitnesspal. https://www .my fitnesspal. com/. Accessed: 202007-10.

[3] Myplate calorie counter. https://www.livestrong.com/ myplate/. Accessed: 2020-07-10.

[4] M. Z. Bayu, H. Arshad, and N. M. Ali. Nutritional information visualization using mobile augmented reality technology. Procedia Technology, 11:396 - 402, 2013. 4th International Conference on Electrical Engineering and Informatics, ICEEI 2013. doi: 10.1016/j.protcy.2013. 12.208

[5] M. Brehmer, B. Lee, P. Isenberg, and E. K. Choe. Visualizing ranges over time on mobile phones: A task-based crowdsourced evaluation. IEEE Transactions on Visualization and Computer Graphics, 25(1):619-629, 2019.

[6] S. Few. Information Dashboard Design: The Effective Visual Communication of Data. O'Reilly Media, Inc., 2006.

[7] D. J. Graham and R. W. Jeffery. Location, location, location: eyetracking evidence that consumers preferentially view prominently positioned nutrition information. Journal of the American Dietetic Association, 111(11):1704-1711, 2011.

[8] C. Mah, R. Pusch, S. Huron, and S. Carpendale. A visualization fingerprint: Comparing nutrient data visually. 2015

[9] N. Pratt. Improving usability of nutrition information through graphical visualization. $\mathrm{PhD}$ thesis, University of Illinois at Urbana-Champaign, 2015.

[10] P. Riehmann, W. Möbus, and B. Froehlich. Visualizing food ingredients for children by utilizing glyph-based characters. In Proceedings of the 2014 International Working Conference on Advanced Visual Interfaces, AVI '14, p. 133-136. Association for Computing Machinery, New York, NY, USA, 2014. doi: 10.1145/2598153.2598203

[11] C. A. Roberto and N. Khandpur. Improving the design of nutrition labels to promote healthier food choices and reasonable portion sizes. International Journal of Obesity, 38(1):S25-S33, 2014.

[12] R. L. Rothman, R. Housam, H. Weiss, D. Davis, R. Gregory, T. Gebretsadik, A. Shintani, and T. A. Elasy. Patient understanding of food labels: The role of literacy and numeracy. American Journal of Preventive Medicine, 31(5):391 - 398, 2006. doi: 10.1016/j.amepre. 2006 07.025

[13] M. Viswanathan and M. Hastak. The role of summary information in facilitating consumers' comprehension of nutrition information. Journal of Public Policy \& Marketing, 21(2):305-318, 2002.

[14] L. Wu, L. Guo, H. Fang, and L. Mou. Bullet graph versus gauges graph: Evaluation human information processing of industrial visualization based on eye-tracking methods. In Advances in Usability, User Experience and Assistive Technology, pp. 752-762. Springer International Publishing, 2019. 